\title{
Using a Functional Ontology of Reputation to Interoperate Different Agent Reputation Models
}

\author{
Sara Casare and Jaime Simão Sichman \\ Intelligent Techniques Laboratory \\ University of São Paulo \\ Av. Prof. Luciano Gualberto, 158 - trav. 3 \\ 05508-900 São Paulo SP BRAZIL \\ \{sara.casare, jaime.sichman\}@poli.usp.br
}

\begin{abstract}
This paper presents a Functional Ontology of Reputation that could be used as a common shared reputation knowledge by agents. Although there is a huge work on agent reputation, each research defines its own basic concepts. Sometimes different meanings are associated to the same term and in other occasions the same meaning is related to different terms. We claim that the reputation knowledge structured as an ontology could be used to enable the semantic integration level involved in the interoperation of software agents using different reputation models. We have illustrated this idea by showing a semantic mapping for the reputation concepts used in three distinct reputation models. That mapping shows how this ontology could act as a common global ontology that supports the semantic integration among these models.
\end{abstract}

Keywords: Reputation, Ontology, Interoperability, Multi-Agent Systems.

\section{INTRODUCTION}

A great number of multi-agent systems (MAS) have been constructed in the last 10 years, with different aims and architectures. In particular, regarding the homogeneity of the agents, in some systems all the agents have the same architecture (homogeneous) and in others agents have different internal models (heterogeneous). In both of them, however, agents share a common communication infrastructure (middleware), like KQML [19, 13].

The advantage of the second approach is enabling the dynamic formation of bottom-up, non-centralized open MAS. We mean by open a MAS where agents may enter and leave dynamically the agent society, without a preestablished central control.

One of the crucial aspects that have enabled the interoperability of such heterogeneous agents systems is a common shared knowledge about key concepts of the field. For instance, even if two or more BDI (Belief, Desire, Intention) agents [24] may have been constructed using different internal architectures, as ASIC (Architecture for Social and Individual Control) [2] and Jason [3], all of them share the same meaning to concepts such as belief and intention.

Although there is a huge work on agent reputation, each research defines its own basic concepts. Sometimes different meanings are associated to the same term and in other occasions the same meaning is related to different terms. For instance, direct reputation [21] and image [10] are examples of the latter, while Direct Trust [27] and Trust [9] are examples of the former. If one wants to build an open multi-agent system, where two different agents, based 
on each of these models, could change their beliefs about reputation, it must be provided a semantic interoperation level that could translate these concepts.

The idea of this work is precisely to cover this gap, i.e., to build a common reputation ontology to represent the key concepts of reputation. This ontology, called the Functional Ontology of Reputation, could be used as a common shared reputation knowledge by agents operating with different reputation models. The goal of this ontology is to put together the broad knowledge about reputation produced in some areas of interest such as psychology and AI, and to represent that knowledge in a structured form. In this way such an ontology could be used to support the semantic interoperation level for agents in a MAS that use different reputation mechanisms.

In order to do so, we must at first compare the several reputation models developed so far, and then extract their main concepts and associated terms. A second step would consist of proposing an architecture for the middleware layer responsible for the semantic interoperation of different models.

This work is organized as follows. In section 2, we briefly present three reputation models as an example of the research in this field. In section 3, we present the main concepts that we believe an ontology of reputation should cover. Based on these concepts, Section 4 outlines a Functional Ontology of Reputation. In section 5, we present the possible approaches to the semantic interoperation level using an ontology for reputative agents. In section 6 , we show a preliminary example of the use of our work as a kind of common global ontology for reputation concepts. Finally, in section 7 we present the current results and future work.

\section{Agent Reputation Models}

After the Oxford Dictionary, reputation is the opinion that people in general have about what somebody or something is. For example, a school may have an excellent reputation whereas a person could have a reputation of being lazy.

Reputation is an indispensable condition for the social conviviality in human societies. The emergence of Internet based virtual societies has caused the migration of reputation related concepts from the world of human interactions to the world of virtual interactions. Reputation and trust systems are key factors for successful electronic commerce environment. They are used as mechanism in order to search trustful partners as well as an incentive to avoid cheaters and frauds [26].
Reputation is a social product as well as a social process. It is a product, or property, in the sense that it consists of opinion agreement in some level; on the other hand, it may be seen as a process in the sense that there is a flow of information and influence in the social network. While reputation as a product may be seen as a cognitive representation (or a belief), reputation as a process consists of a set of beliefs' transmission in the social network $[6,10]$.

In the last years several computational models of reputation have been proposed $[21,26,31,32$ among others]. However an analysis of these models indicates that sometimes the notion of reputation is used in an intuitive way. In most cases they use neither a precise definition of reputation nor the theoretical or empiric bases from disciplines that have worked with reputation concepts much longer than Artificial Intelligence (AI), such as Economy, Sociology and Psychology $[6,12]$.

As an example of research produced in the MAS field we describe three of these models in this section: a cognitive reputation model, a typology of reputation and the reputation model used in the Regret system. The specifications of these models are in different degrees of computational formalism. The cognitive reputation model [10] consists of a high level conceptual model, described in natural language. The typology of reputation [21] is a taxonomy regarding reputation sources of information, while the ReGret system [27, 25] is a computational mechanism that involves a reputation model in its kernel.

These models, together with the Functional Ontology of Reputation ontology proposed in section 4, have been used in the example of integration among software agents using different reputation models, as shown in section 6. However, before presenting this integration, we need to give a brief description of each of these models.

\subsection{Cognitive Reputation Model}

The Cognitive Reputation Model proposed by Conte and Paolucci [10] treats the several aspects associated with the reputation transmission in the social sphere. Image and reputation are two central concepts in this model. Image is an evaluative belief, as "good" or "bad", formed using information acquired by agent experience and reflects the target agent behavior.

Reputation is a belief about others' minds, a metabelief that results from beliefs transmission. It represents the process as well as the effect of transmission of a target image.

Besides, the model describes the concepts related 
to the set of agents involved in image and reputation formation and transmission. Target agents are individuals, groups, or even artifacts that play the role of the evaluation object. Evaluator agents are those entities able to develop an evaluative belief about others as an effect of their social interactions and perceptions. Propagators, or third parties, are those entities able to transmit reputation information about a target to another entity beneficiary agents. Beneficiaries are individuals, groups, or organizations for which the evaluation of the target brings some benefit. As the authors emphasize, there is a non-empty intersection between these four sets of agents. Therefore, an agent can be at the same time a member of the evaluators set as well as a member of the propagator and the beneficiaries' sets.

Figure 1 shows the image formation based on agent experience, the reputation diffusion and the contagion provoked by reputation presence, that may reinforce the initial experience.

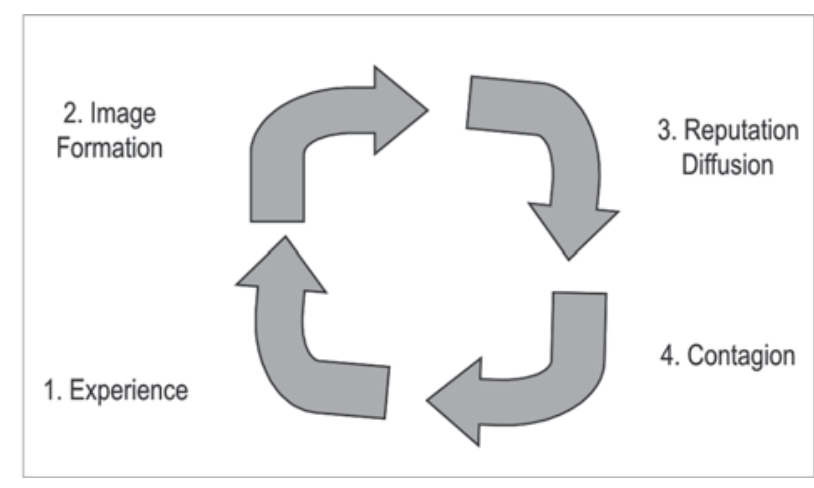

Figure 1: Image and Reputation in the cognitive model of reputation [10]

\subsection{Typology of Reputation}

The Typology of Reputation proposed by Mui et al. [21] is based on the reputation research done by several areas of interest, such as Economy, Computer Science and Biology. This typology distinguishes reputation notions in three classification levels, as we can see in figure 2 . The first level distinguishes reputation according to the target entity nature, while the second and third levels distinguish individual reputation according to the information source.

In the first level there are two concepts:

- Individual reputation is the reputation that can be used to describe an individual;

- Group reputation is reputation that can be used to describe a group of individuals.

Figure 2 shows a representation of this typology using Unified Model Language (UML) class diagram.

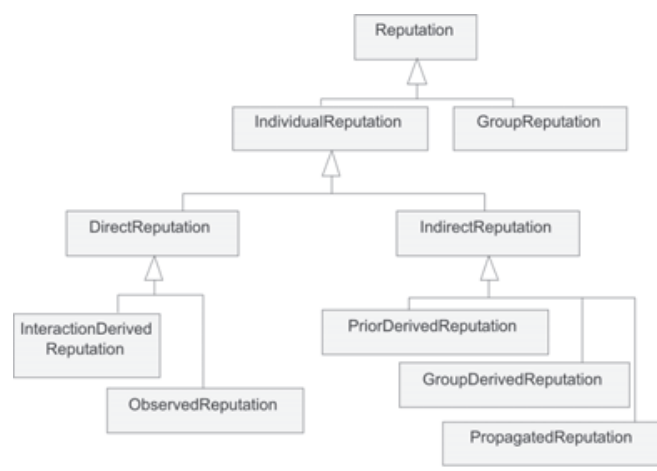

Figure 2: Reputation typology proposed by Mui et al. [21]

The second and third level contains the following concepts:

- Direct reputation is the reputation derived from direct encounter or observation. It has two sub concepts: interaction derived reputation, the one based on actual encounters between a target agent and its evaluating agent; observed reputation, that one resulting from observations made about another agent's encounters;

- Indirect Reputation is the reputation based on second-hand evidence. It has three sub concepts: group derived reputation that is estimates for agents in social groups; prior derived reputation, based on prior beliefs about strangers; propagated reputation, based on information gathered from others.

\subsection{ReGret System}

The ReGret system [25, 27] is a trust and reputation system developed for complex e-commerce environments where social relationships play an important role. The idea is to consider the social aspects involved in the reputation and trust notions, taking into account the social structures among the members of a society in the evaluation of these notions. This model deals with four kinds of reputation evaluation: Direct Trust, Witness Reputation, Neighbourhood Reputation, System Reputation.

- Direct Trust refers to the evaluation that is built from direct interaction, including both direct experience and direct observation;

- Witness Reputation is calculated using the information gathered from other agents of the community that had direct experience with the target agent;

- Neighbourhood Reputation is the reputation based on social prejudice;

- System Reputation is the reputation based on agent membership to a certain group. 
Additionally, the Regret system proposes the Credibility notion, a kind of rating associated to the received information about reputation that evaluates the truthfulness of information received from a witness. The trust evaluation is the result of these reputation evaluation, as shown in figure 3 .

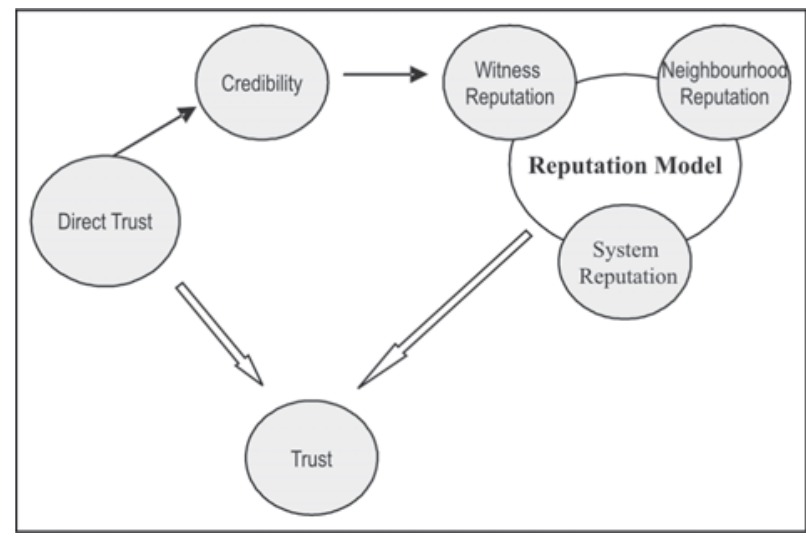

Figure 3: Reputation Concepts in Regret System [27]

Once described these agent reputation models, we are ready to present the main concepts we believe that are essential for an ontology of reputation that should be used by autonomous agents, as presented next.

\section{Reputation Notion}

This section defines the concepts used to identify the several aspects of a reputation, both as a product and as a process, as we can see in figure 4: reputation nature, roles involved in reputation formation and propagation, information sources for reputation, evaluation of reputation, and reputation maintenance. Those aspects constitute a set of ontological terms used in a Functional Ontology of Reputation, presented in section 4. For more details see [7].

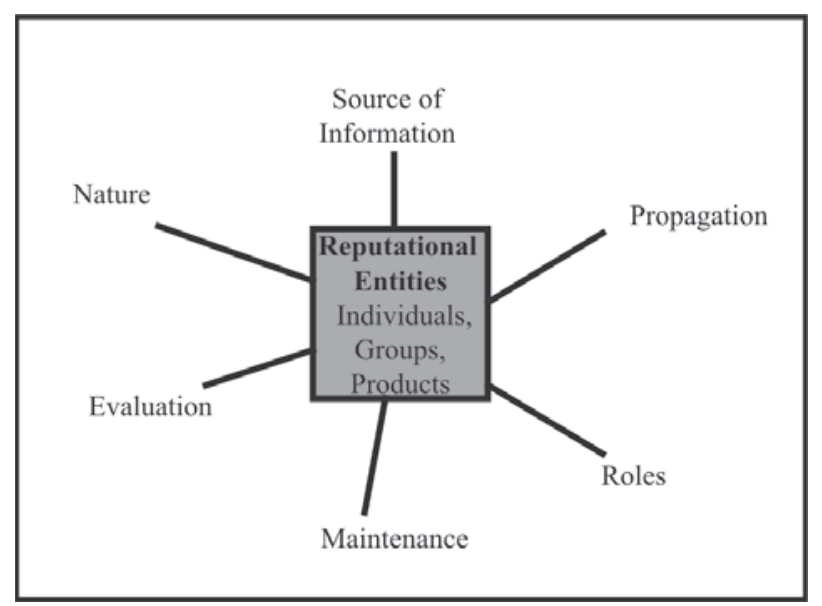

Figure 4: Several aspects of a reputation inspired by Bromley[6]

\subsection{Reputation Nature and Roles}

The nature of the reputation distinguishes a reputation according to the kind of entity it is associated to. According to Bromley [6] there are different sorts of reputational entities, given that persons, groups of people and corporations can have a reputation, as well as products, services, geographical areas, and events in a general way, including activities.

Reputation formation and propagation involves several roles, played by the entities or agents that participate in those processes, such as target role and evaluator role.

\subsection{Information Source of Reputation}

Reputation, as part of collective systems of beliefs and opinions about people and things [6] can be classified according to the origin of these beliefs and opinions. Beliefs can derive from several sources, such as direct experiences, received information, and social group prejudices.

\subsection{Reputation Evaluation and Measurement}

A reputation can be summarized in a word or in an expression, such as "good" or "very good", or it can be detailed through examples and justifications. In general, a reputation can be evaluated through several types of measurements and different factors [6].

The measurement types that can be employed in the evaluation of a reputation allow different levels of detail, as in a level of esteem such as "bad", "good" and "great", or a more specific evaluation that considers the content of a reputation. That content evaluation allows identifying which are the aspects involved in a reputation as well as the relevance given to each one of them.

\subsection{Reputation Maintenance}

The reputation maintenance process deals with the modifications that occur in the content and structure of a reputation over time. Temporal aspects play an important role in the process of maintenance of a reputation. Some authors $[12,32]$ consider that the most recent behavior should weight more in a reputation maintenance process than the oldest ones, since a reputation should reflect the agent's current behavior in spite of older ones.

\subsection{Reputation Propagation}

A reputation propagation process deals with various aspects involved in transmission of reputation. Information related to reputation is not just transmitted by the propagator entity, which, instead, selects, invents and manipulates the original information according to the restrictions and opportunities presented by the circumstances $[6,10,27]$. 


\section{Functional Ontology of Reputation}

This section outlines a Functional Ontology of Reputation. The goal of this ontology is to put together the broad knowledge about reputation produced in some areas of interest such as Psychology and Artificial Intelligence, and to represent that knowledge in a structured form.

The term Ontology originally designates a philosophy branch dealing with the a priori nature of the reality [16]. Although there are many definitions of ontology, the more popular is that proposed by Gruber [15]: an ontology consists of an explicit specification of a conceptualization, where a conceptualization is an abstract view of the world that one desires to represent, consisting of objects, concepts and the relations among them. The specification of a conceptualization comprises both the description of these world entities in natural language and the creation of the axioms that refine objects, concepts and relation definitions.

Ushold and Gruniger [28] proposes a simple answer to the question "What is an ontology": it designates the shared understanding of some domain of interest and entails a world view of that domain. This world view is often represented as a set of concepts, such as entities and attributes, their definitions and their relationship, which constitutes a conceptualization.

\subsection{Functional Ontologies}

The Functional Ontology of Reputation proposed in this paper employs the knowledge categories proposed by Valente [4, 29] as part of his work on Legal Knowledge Engineering, called the Functional Ontology of Law.

As its name indicates, this ontology adopts a functional perspective, that means, the law is interpreted and analyzed through a functional point of view. This perspective can be described as follows [4]:

- The legal system as a whole, and also each of its components, exits to execute determined functions in order to achieve a social goals;

- Given that the main function of the legal system is to react to social behavior, it can be viewed as a kind of social device operating within / on society to regulate social behavior;

- The functions that distinguish different types of legal knowledge can be described in two ways. First, they point out the legal system subfunctions. Second, they divide legal knowledge into a set of basic categories which provide support for legal functions.

\subsubsection{Functional Ontology of Law}

This ontology is basically a set of interconnected primitive categories and sub-categories of legal knowledge, distinguished through a functional perspective. These categories are: Normative Knowledge, World Knowledge, Responsibility Knowledge, Reactive Knowledge, Creative Knowledge, Meta-legal Knowledge.

The Normative Knowledge is the most characteristic category of legal knowledge and has two functions: prescribing behavior and defining a standard of comparison for the social reality.

The World Knowledge is a kind of interface between the real world and the legal one. Its role is to define a model of the real world which is used as a basis to express the other categories of the legal system.

The Responsibility Knowledge has as function the assignment or limitation of the responsibility of an agent in a given situation. In that way it is possible to establish a link between the violation of a norm and the agent that is considered responsible for this violation.

The Reactive Knowledge is responsible for the reaction towards the agent assigned responsible for an illegal situation, specifying which legal sanction should be taken against that agent.

The Creative Knowledge is responsible for creating legal entities that did not exist before as part of legal world, like the creation of a department within the government.

Finally, the Meta-legal knowledge comprises two main functions: (i) to implement the dynamics of the legal system using what is called validity knowledge, and (ii) to institute mechanisms to solve conflicts between instances of all categories of legal knowledge.

\subsubsection{From Legal to Social World}

While in the legal world a rule violation generates a legal punishment for the infringing agent, in the social world the penalty associated to an agent that violates a social norm is a bad reputation [8].

Given that we claim that the concepts of the legal world can be used to model the social world, through (i) the extension of the concept of legal rule to social norm, (ii) the internalization of social control mechanisms in the agent's mind, so far externalized in legal institutions, and (iii) the replacement of the legal sanction to a social one, i.e., a reputation.

As in the Functional Ontology of Law [29], the distinction among the categories of the Functional Ontology of Reputation will be accomplished according to a functional perspective, in which each component of 
the reputation system, embedded in the social system, exists to perform a specific function in the effort to achieve social objectives, such as trust, reciprocity and social cooperation.

\subsection{Main Categories of Functional Ontology of Reputation}

The Functional Ontology of Reputation includes, as a kernel, the reputation concepts presented in section 3. That kernel is embedded in a broad framework, composed by the knowledge categories inspired by the Functional Ontology of Law. In that way we have outlined the whole mechanism related to reputation notion in the agent's mind.

This ontology has been implemented in Ontology Web Language - Description Logic (OWL DL) [11] using Protégé [18] as an ontology-modeling environment. OWL language was designed to meet the requirements of a Web Ontology Language and is part of the W3C (World Wide Web Consortium) recommendation related to the Semantic Web. The Semantic Web [1] is a future vision of the Web, where information will have explicit meaning, making it easier for computers automatically integrate and process information available on the Web.

An OWL DL ontology consists of class, properties and individuals. Classes, interpreted as sets of individuals, are defined using formal description that states the requirements for membership of a class. Classes can be organized into superclass - subclass hierarchy. Subclasses specialize (or is subsumed by) their superclasses. Individuals represent objects in the domain we are interested in, while properties are relations that link two individuals. Two types of properties can be distinguished: (i) object properties, that are relations between instances of two classes; (ii) datatype properties, that are relations between instances of classes and RDF literals or XML Schemas datatype. One of the key features of an ontology described using OWL DL is that we can reason over it, using a reasoner programs such as RACER [17] to infer over the ontology.

The Functional Ontology of Reputation contains four main categories: Reputative Knowledge, Responsibility Knowledge, Normative Knowledge and World Knowledge. Besides we have defined Common Knowledge category in order to represent concepts related to the common sense that in some way are related to Reputative Knowledge. This ontology contains 85 classes and 40 properties. As we can see in figure 7, these classes are divided into two main knowledge categories: 67 in Reputation Knowledge category and
18 in Common Knowledge category.

Each of these five main categories is detailed in the next sections.

\subsubsection{Common Knowledge}

Common Knowledge category represents the concepts related to the common sense that in some way are related to Reputative Knowledge. It is worthy noting that common sense ontologies is a research field in its own [5]. However the goal of this category is to define common sense concepts that are directly involved with reputation notion.

This category was borrowed from the Functional Ontology of Law and extended in order to include information sources of reputation as well as reputational entities. Additionally, it includes the time concept. Information source represents those facts that act as a source of information for a reputation definition, such as direct experience, group characteristics, propagated information, observation of behavior and prejudice.

Reputational Entities represent all things that are able to play at least one reputative role and then take part in a reputation process, such as individuals (agents or human being), group of individuals, objects and services.

\subsubsection{Reputative Knowledge}

Reputative Knowledge is the most characteristic category in the Functional Ontology of Reputation, involving 45 classes. It was inspired by the Reactive Knowledge from the Functional Ontology of Law [29]. Its main function is to deal with the reputational entity reward (good reputation) or penalty (bad reputation), according to its behavior. This category models the products as well as the processes involved in the reputation notion, described in section 3 .

\section{Reputation Roles}

Reputation Role concept represents those roles played by entities involved in reputative processes, such as reputation evaluation and reputation propagation. Putting together the target notion proposed by Conte and Paolucci [10] and the reputational entity notion proposed by Bromley [6], we defined the target role, played by all of the entities that act as reputation object, such as people (individuals or groups), things, products and service, places, events and activities.

Based on the evaluator, propagator and beneficiary sets of agents [10] we defined the evaluator role, the propagator role and the recipient role, respectively. The recipient role extends the notion of 
beneficiary set of agent in the sense that it makes explicit the action of receiving information about reputation related to the action of transmitting it. These four roles can be played by persons or groups of people, as in daily life, or by software agents, as in online systems, such as e-commerce systems and electronic chat systems.

\section{Reputation Property}

Reputation Property concept represents two main reputation dimensions: reputation nature and reputation type, as shown in figure 5 .

Reputation Nature concept distinguishes reputation according to the nature of a reputational entity: individual reputation, related to persons or individual agents; group reputation, related to the individuals or agent groups; product reputation, associated to products and services; location reputation, associated to geographical places; event reputation, associated to events in a general way; and activity reputation, related to the activities executed by agents.

Reputation Type concept distinguishes a reputation according to information source used in its formation. We adopted the term primary reputation to designate the reputation resulting from the primary sources of information. We called direct reputation the one derived from direct encounters among agents. We used observed reputation to refer to a reputation derived from the observation of those interactions.

We adopted the term secondary reputation to name the reputation resulting from the secondary sources of information. We adopted the term proposed by Mui et al. [21], propagated reputation, to designate the one derived from received information. We called collective reputation the one agent inherit from their social group, and stereotyped reputation the one based on social prejudices.

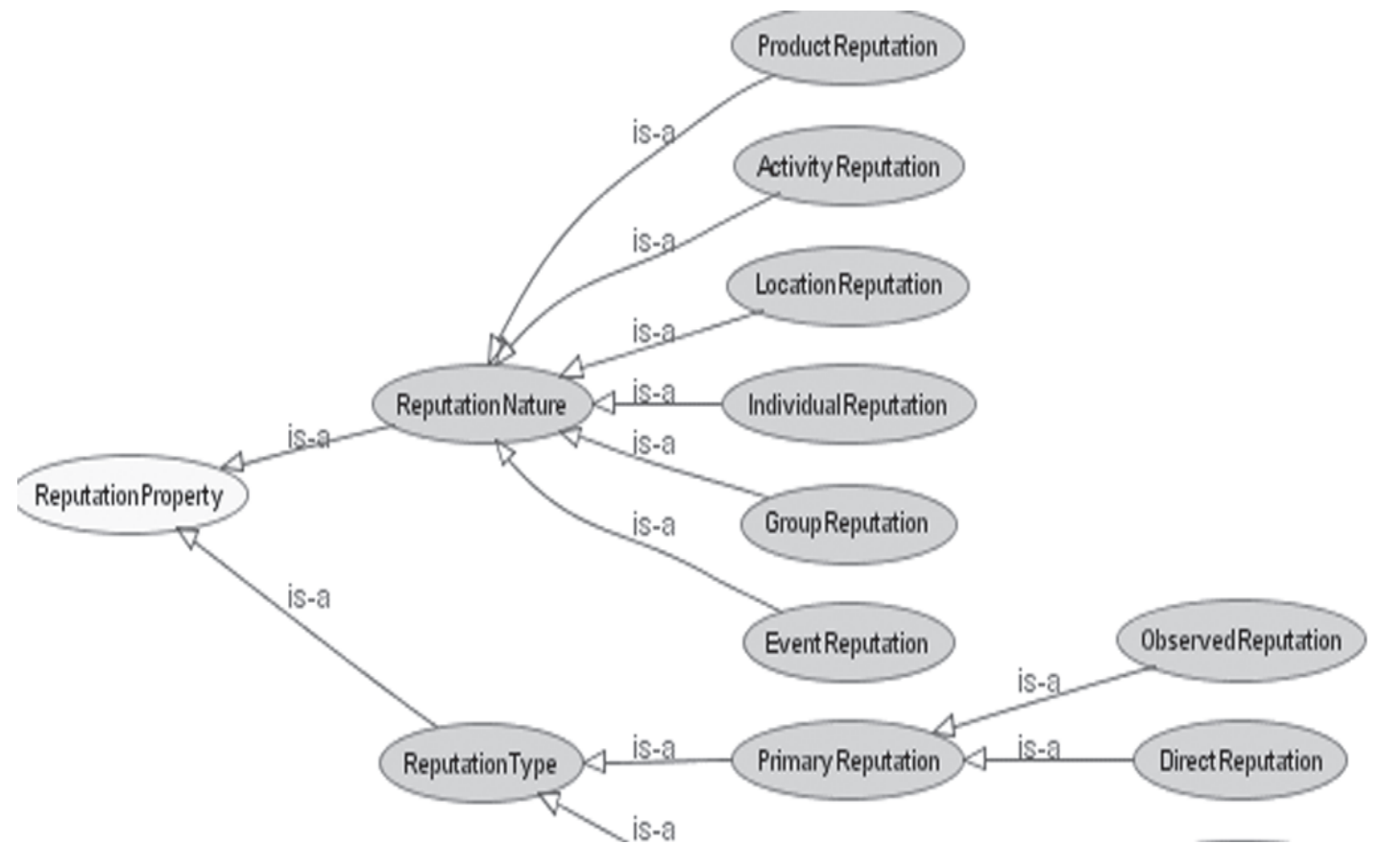

Figure 5: Reputation Property representation

\section{Reputation Evaluation Components}

Reputation Evaluation Component represents those aspects taken into account during the establishment of a reputation value.

Evaluation Factor concept represents the four factors that somehow have influence on a reputation evaluation. We adopted the following terms in order to identify these factors: Target Behavior, Evaluator

\section{Reputation, Propagator Reputation and Target Evaluator} Social Relationship.

Reputation Evaluation Value represents the worth of a reputation after an evaluation process. It can be expressed as a qualitative measure ("good", "bad"), or as a quantitative measure $(0,1,2,10)$. Reputation Final Value represents the worth of a reputation after a maintenance process. Such as Reputation Evaluation Value it can be expressed as a qualitative or a quantitative measure. 
Evaluation Attribute represents the detailed aspects of a reputation evaluation. For instance, one can consider price, quality and durability when evaluates a product reputation. Evaluation Attribute Weight represents the importance an evaluator entity associates to each one of reputation attributes. For example, price attribute is more important than quality attribute.

\section{Reputation Process}

Reputation Process concept represents the four processes involved in reputation notion: Reputation Reception, Reputation Transmission, Reputation Evaluation and Reputation Maintenance.

Reputation Transmission Process represents the process that deals with passing reputation information from one agent to another in a social network, while Reputation Reception Process represents the process that deals with getting or accepting reputation information from one agent.

Reputation Evaluation Process represents the process of measuring a reputation. The two measurement types that can be employed in such process are represented as Reputation Evaluation Process subclasses. Reputation Content Evaluation Process represents a reputation measurement process that deals with the attributes that contribute in its composition as well as the relevance (or weight) associated to them. It permits to describe a reputation value in terms of attributes and their respectively weights. Reputation Esteem Level Evaluation represents a reputation measurement process that produces a reputation value that cannot be described in details. Both processes can produce qualitative or quantitative reputation value.

Reputation Maintenance Process represents the process that deal with reputation alterations over time. That concept involves two subclasses, aggregation process and historical process. We called Aggregation Process the one that deals with impact on reputation value caused by evaluation of agent's current behavior: a positive impact improves reputation; a neutral impact maintains the same reputation; a negative impact decreases reputation. We called Historical Process that one in charge of historical dimension of reputation. That process specifies the historical extension of previous evaluations considered as well as the relevance associated to the most recent evaluations in spite of oldest ones.

\subsubsection{Responsibility KNOWLEDGe}

Responsibility Knowledge category was borrowed from the Functional Ontology of Law [29] and extended to deal with behavior causes. Its main function is to associate a cause to a specific behavior, in order to define whether the reputational entity must be considered responsible for this behavior or, instead, there are circumstances that attenuate and restrict its responsibility. Responsibility Knowledge represents two main notions, namely, the attribution notion and the actor-observer effect notion, and involves six classes.

\section{Attribution and related classes}

There are five classes related to attribution notion: Attribution, Internal Cause, External Cause, Responsibility Assignment and Responsibility Restriction.

Attribution is a term used in social cognition to refer to the causes for which the actions are interpreted in terms of personal characteristics (internal cause) or external circumstances (external cause), in order to provide a causal explanation for the associated behaviors [6]. Attribution processes are part of our inclination to impose a pattern of meaning to observed facts. Concerning reputation, one of major attribution effects is to give consistence and coherence to agent behaviors.

A behavior has Internal Cause when interpreted as depending exclusively on the agent, for instance, agent's motivation or agent's capacity. A behavior has External Cause when considered as depending exclusively on external circumstances, such as opportunities or threats [6].

Responsibility Assignment defines that a reputational entity should be held responsible for its behavior, given that it occurred under a condition interpreted as depending on internal causes. Responsibility Restriction defines that a reputational entity cannot be held responsible for its behavior, given that it occurred under certain condition interpreted as depending exclusively on external cause instead of internal cause.

\section{Actor-Observer Effect}

Attribution process can be affected by the socalled Actor-Observer Effect. That term is used to designate the tendency to attribute internal causes to other people's behavior and to attribute external causes to one's own behavior. Therefore, that effect can provoke a fundamental attribution mistake. Bromley [6] points out that the actor-observer effect is most likely to affect our perception of people we know least well than of people we know better well. The Actor-Observer Effect class represents this notion. 


\subsubsection{Normative Knowledge}

Normative Knowledge category was borrowed from the Functional Ontology of Law and extended to deal with social norms instead of legal rules. Its main function is to prescribe the agent behavior, through the description of the social norms. The idea is to compare the agent actual behavior with the ideal one, prescribed by the norms, and then conclude whether the behavior is adequate or inadequate. That category contains 7 classes, where Social Norm and Normative Status are the main ones.

Social Norm represents norms that are valid in a society and corresponds to an extension of legal norm concept from Functional Ontology of Law. Social norms can be specified as Positive Norm, when describing an adequate behavior, or as Negative Norm, when describing an inadequate behavior. For example, "Allowed to smoke in open places" is an instance of positive norm, while "Forbidden to smoke in closed places" is an instance of negative norms while.

Normative Status represents a normative classification of the actual behavior of an agent, after being compared to the social norms. This concept has three subclasses: Adequate Status, Inadequate Status and Neutral Status. Adequate Status is associated to a positive norm and classifies an actual behavior as adequate, while Inadequate Status is linked to a negative norm and classifies the agent behavior as inadequate. Finally, the Neutral Status classifies actual behaviors that are not described through social norms.

\subsubsection{World KNOWLEdGe}

World Knowledge category was borrowed from the Functional Ontology of Law and adapted to represent the world knowledge that are relevant to Reputative Knowledge. Its main function is to provide a model of social world which is used by the other categories in order to encapsulate common sense notions.

That category involves the following classes, Reputation Concept, Reputation Relation, and Case, that correspond respectively to the concepts Legal Concept, Legal Relation and Case of the Functional Ontology of Law. Reputation Concept represents the class of things in the world, while Reputation Relation depicts the relations that exist between reputation concepts. Case represents a reputative entity behavior in a given circumstance. Cases can be compared to social norms in order to define the normative status associated to them.

\subsection{Functional Ontology of Reputation Overall View}

A big picture of the relation among the Reputation knowledge categories of the Functional Ontology of Reputation is shown in figure 6, inspired by Valente [29].

The agent society as well as agents' behavior is represented by the inferior rectangle, while the superior rectangle represents the reputative system within an agent's mind.

A cycle starts with the interpretation of the agent social behavior by the World Knowledge category. This category describes the agent behavior in terms of social norms as well as identifies the agent associated to the behavior.

After this first step, Normative Knowledge category receives this behavior description and matches it with an positive social norm or a negative one, generating the correspondent normative status, Adequate or Inadequate, respectively.

Responsibility Knowledge category then defines whether the agent must be held responsible for its behavior (responsibility assignment) or not (responsibility restriction).

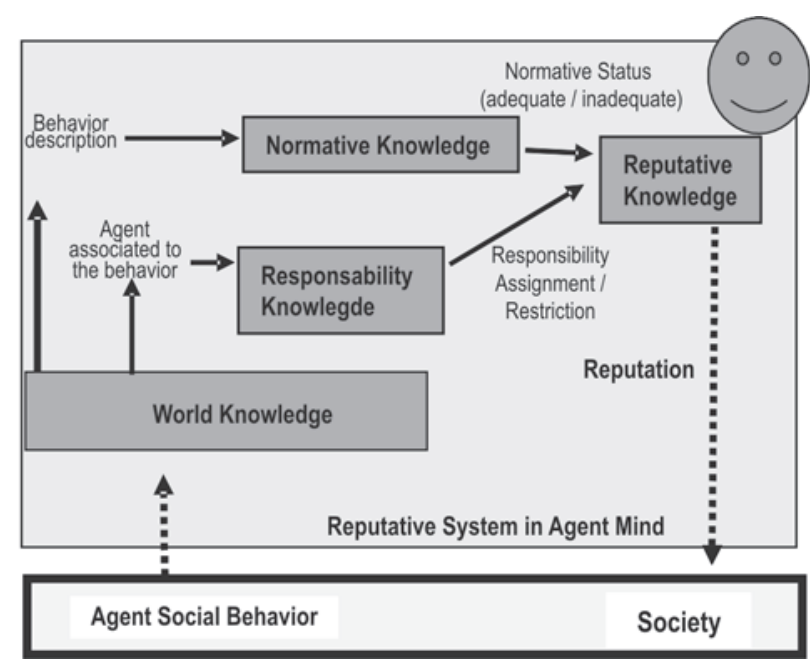

Figure 6: Categories of a Functional Ontology of Reputation

Finally, using the normative status and the responsibility information Reputative Knowledge category is able to define the agent reputation, as a reward (good reputation) or a penalty (bad reputation).

Figure 7 shows a class diagram that depicts the first five layers of the Functional Ontology of Reputation. 


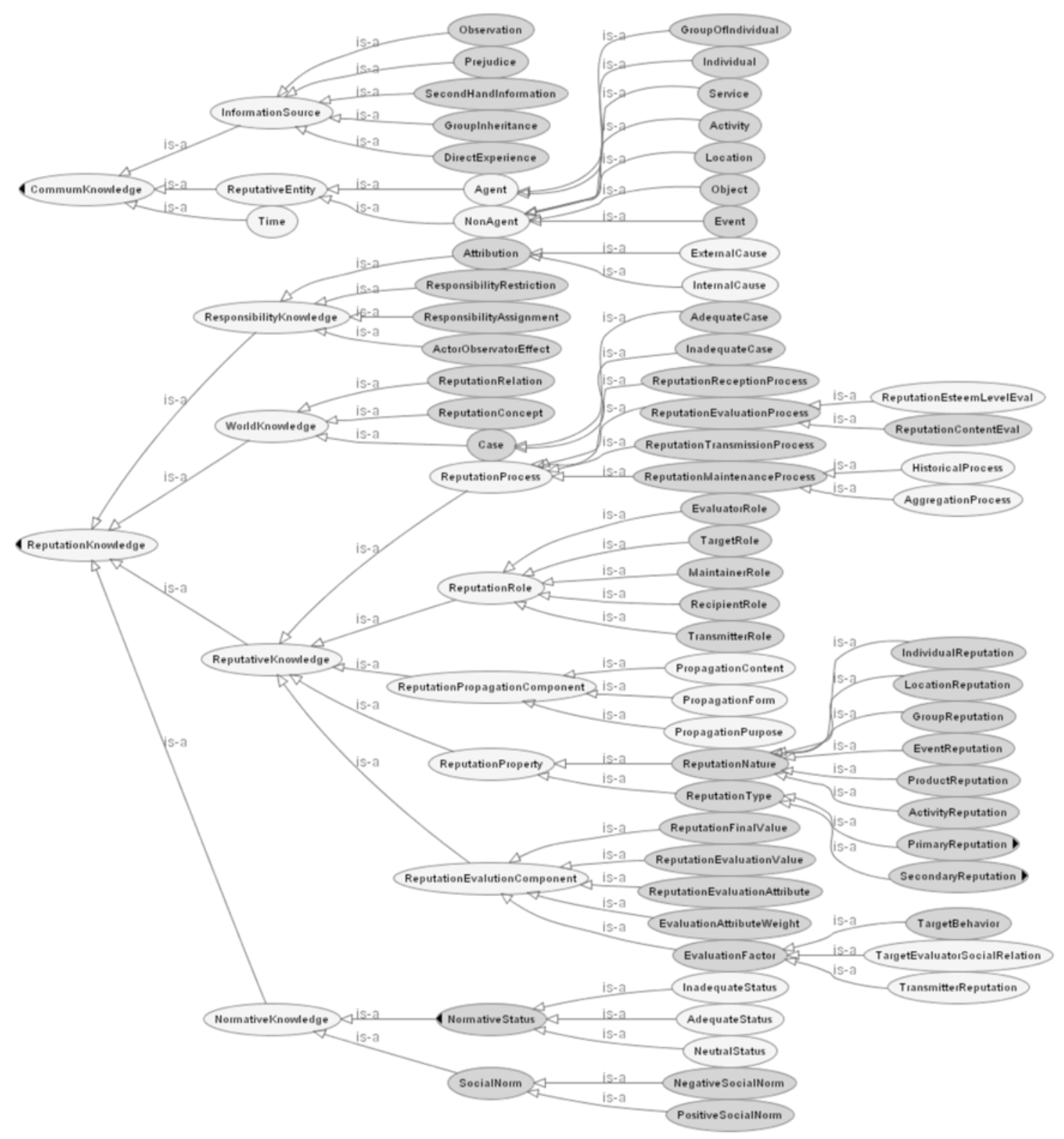

Figure 7: First five layers of Functional Ontology of Reputation

Figure 7 is an almost complete picture of the ontology, since just two classes have their subclasses hidden: Primary Reputation and Secondary Reputation. See figure 5 for more details about these classes.

Once defined the classes and relations of the Functional Ontology of Reputation, we describe in the next two sections how such an ontology could be used to enable heterogeneous agents (that use different reputation models) to interoperate and change their impressions about the other agents in the system.

\section{POSSIBLE APPROACHES FOR THE INTEROPERATION of Reputation Models}

According to Uschold and Gruninger [28] the space of use for ontologies can be divided in three categories:

- communication between people, organization and software systems, enabling shared understanding;

- inter-operability in environment such as multiagent system, where there are distinct languages and knowledge representation, allowing the exchange of information as a kind of inter lingua; 
- systems engineering, providing support to the design and development of the software systems.

Visser et al [30] suggest that interoperation among different system involves three levels of information source integration: syntactic integration, structural integration and semantic integration.

Syntactic integration can be achieved using standards like HTML and XML, while structural integration, that involves re-formatting data structures to a new homogeneous one, can be solved by a middleware like CORBA.

Semantic integration is more complex than the formers, given the heterogeneity of concepts meaning. It can be achieved applying ontologies to construct a semantic mapping involving shared vocabulary and explicit conceptualizations.

Visser et al [30] propose that ontologies can be used to support semantic integration of heterogeneous sources of information. According to these authors, this integration may be implemented in three different ways:

- A centralized approach, where each source of information is related to one common domain ontology. This approach has a low scalability, given that the global ontology contains all domain terms, and each information source is related to it;

- A decentralized approach, where every source of information is related to its own ontology. In such approach it is assumed that a pre-defined ontology exists for each source. The pros consist in the ease way that new sources can be added or removed. The cons are related to the comparison of heterogeneous ontologies that use their own vocabulary, rising lots of synonyms, homonyms and loss of information. This approach requires the use of ontology alignment tools like OBSERVER [20] and PROMPT [22];

An Hybrid approach, where every source of information has its own ontology and the vocabulary of these ontologies are related to a common ontology. That ontology organizes the common global vocabulary in order to support the source ontologies comparison. This approach offers flexibility, given that new sources can be easily integrated and comparable to the others. In contrast with to the decentralized approach, the source ontologies remain comparable.

Figures 8, 9 and 10 show examples of how these approaches can be applied in the context of MAS, where agents use the three different reputation models presented in section 2: (A) the cognitive reputation model proposed by Conte and Paolucci [10], (B) the typology of reputation proposed by Mui et al. [21], (C) the reputation model proposed in the ReGret system [27].

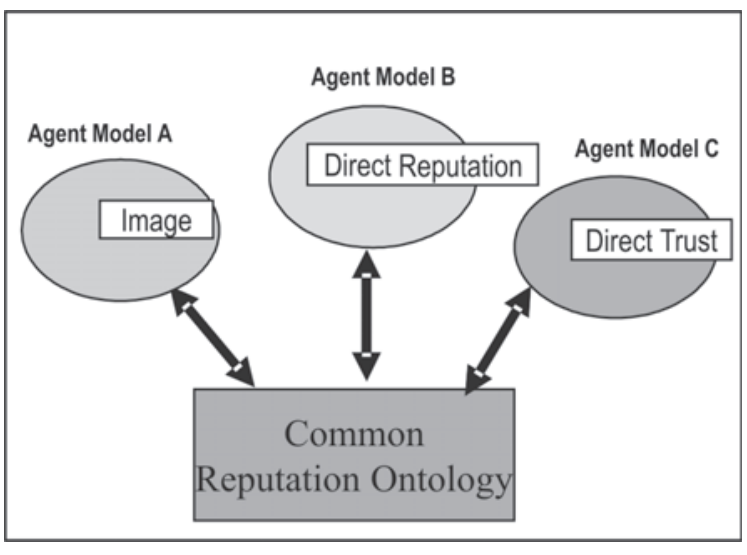

Figure 8: Centralized approach applied in MAS

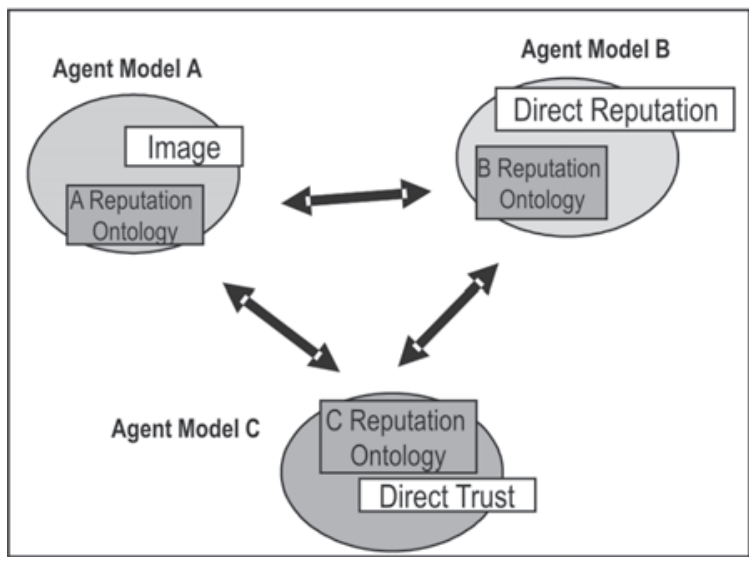

Figure 9: Decentralized approach applied in MAS

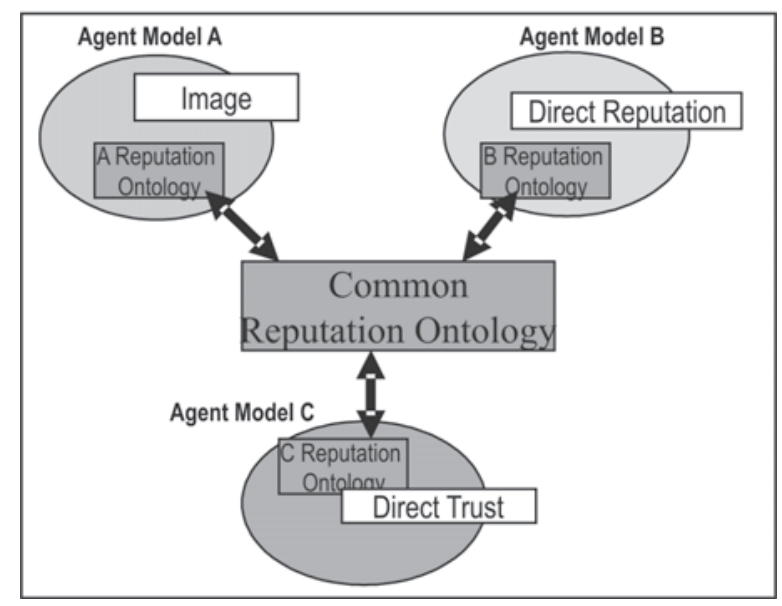

Figure 10: Hybrid approach applied in MAS

Applying these three approaches for semantic integration in order to solve our problem, i.e., the interoperation of different reputation models, we have the following issues:

(i) the adoption of the centralized approach is not an adequate choice, since it would limit the current diversity of 
different agents reputation models and the main idea is not to create a single reputation model. Moreover, whenever a new agent enters the society, the global ontology would have to be expanded and the other agents would have to take these new concepts into account, leading to scalability issues;

(ii) on the other hand, a pure decentralized approach would lead to a different problem. Since a global more abstract ontology is not created, one should provide for each of $n$ different reputation models (n-1) alignment processes in order to enable interoperation. Moreover, as these different models may use concepts that are not considered in other models, the quantity of loss of information due to alignment procedure would be possibly very high, compromising the very goal of interoperation.

(iii) the hybrid approach offers a good tradeoff as a solution to the semantic integration of reputation models. In one hand, it does not limit reputation models diversity in an heterogeneous MAS environment, given that each model can operates with it own ontology. In the other hand, it does not involves complex ontology alignment procedure because the vocabulary of these different ontologies are related to a common global reputation ontology.

Given that, ontologies can be applied in multi-agent systems based on distinct architectures, where different agent must communicate in order to achieve their goals. The Functional Ontology of Reputation presented in section 4 can be used for such purpose, as we can see in the example described in the next section. In this example we have applied the hybrid approach for the semantic integration of reputation models, where the Functional Ontology of Reputation plays the role of a common global ontology.

Additionally, we suppose that every agent is able to communicate with the others using KQML [19] or FIPA [13] standards, i.e., there is a common and shared standard for agents' communication. Moreover, we suppose that each agent's internal architecture can deal with reputation knowledge. It is out of scope of this paper to detail more deeply these architectural options. Our interest is to show that the Functional Ontology of Reputation contains sufficient knowledge to act as a common global ontology since it represents concepts used in other reputation models, as shown next.

\section{EXAMPLE OF INTEROPERATION OF REPUTATION Models}

As we have seen in section 4 the Functional Ontology of Reputation has been implemented in OWL DL language using Protégé [18] as ontology modeling environment. We have used RACER [17] as reasoner to infer over the ontology in order to produce the example shown in this section. RACER can execute the subsumption test that determines whether a class is a subclass of another class. Such functionality generates an inferred ontology class hierarchy. In Protegé the 'manually defined' class hierarchy is called the 'asserted hierarchy', while the class hierarchy automatically computed by the reasoner is called the 'inferred hierarchy', as we can see in figure 11. The task of computing the inferred class hierarchy is also known as classifying the ontology.

The idea is to use the Functional Ontology of Reputation as a common ontology in order to support semantic integration among software agents using different reputation models. As example we have selected the main concepts involved with reputation notion of the three reputation models described in section 2 and aligned them in terms of Functional Ontology of Reputation concepts. These models are the Cognitive Reputation Model proposed by Conte and Paolucci [10], the Typology of Reputation proposed by Mui et al. [21] and the reputation model defined in the ReGret system proposed by Sabater [27].

The following three steps enable the semantic mapping of reputation concepts in terms of the Functional Ontology of Reputation:

1. Gathering information about the reputation model to be integrated, in order to identify the main concepts related to reputation.

Taking the ReGret system as working example, we have identified the following reputation related concept:

- Direct Trust,

- Witness Reputation,

- Neighbourhood Reputation,

- System Reputation,

- Credibility.

2.Defining these concepts in OWL DL in order to form an ontology, and relating the vocabulary of these ontologies to the common ontology, the Functional Ontology of Reputation.

This step consists indeed in creating an ontology for each model, since these reputation models are not described in ontological terms. This task can be supported by ontology alignment tools like PROMPT [22] in order to accelerate the process of relating the vocabulary. However it should involve a human operator who makes the final decision about whether to accept, edit or reject the alignment produced by the tool [30].

In this example we have not used such a tool. Instead, we have done manually the definition of reputation model concept in terms of the Functional Ontology of Reputation. 
It is worthy noting that the goal of this example is to show that this ontology can be used as a common global ontology for reputation concepts and not to demonstrate how we can apply alignment tools in order to accelerate this task.

In our working example, this step produces the description of Regret system concepts using the vocabulary of the Functional Ontology of Reputation. Table 1 shows this result.

Table 1: Regret concepts using common vocabulary

\begin{tabular}{|l|l|}
\hline $\begin{array}{l}\text { Regret Con- } \\
\text { cept }\end{array}$ & $\begin{array}{l}\text { Regret concept description using } \\
\text { common ontology vocabulary }\end{array}$ \\
\hline Direct Trust & $\begin{array}{l}\text { The instances of Direct Trust class } \\
\text { have at least one association } \\
\text { (existential quantifier) through } \\
\text { property has/nformationSource to } \\
\text { instances of Direct Experience class } \\
\text { or Observation class: } \exists \text { hasInforma- } \\
\text { tionSource (DirectExperience } \\
\cup \text { Observation) }\end{array}$ \\
\hline $\begin{array}{l}\text { Witness Repu- } \\
\text { tation }\end{array}$ & $\begin{array}{l}\text { The instances of Witness Reputation } \\
\text { class have at least one associa- } \\
\text { tion through property has/nforma- } \\
\text { tionSource to instances of Propagat- } \\
\text { edlnformation class: } \exists \text { hasInformation- } \\
\text { Source Propagated Information }\end{array}$ \\
\hline $\begin{array}{l}\text { Neighbourhood } \\
\text { Reputation }\end{array}$ & $\begin{array}{l}\text { The instances of this class have } \\
\text { at least one association through } \\
\text { property hasInformationSource to } \\
\text { instances of Prejudice class: } \exists \\
\text { hasInformationSource Prejudice }\end{array}$ \\
\hline $\begin{array}{l}\text { System Repu- } \\
\text { tation }\end{array}$ & $\begin{array}{l}\text { The instances of this class have } \\
\text { at least one association through } \\
\text { property has/nformationSource to } \\
\text { instances of Group Characteristic } \\
\text { class: } \exists \text { hasInformationSource Group- } \\
\text { Characteristic }\end{array}$ \\
\hline
\end{tabular}

\begin{tabular}{|l|l|}
\hline $\begin{array}{l}\text { Regret Con- } \\
\text { cept }\end{array}$ & $\begin{array}{l}\text { Regret concept description using } \\
\text { common ontology vocabulary }\end{array}$ \\
\hline Credibility & $\begin{array}{l}\text { The instances of this class have } \\
\text { at least one association through } \\
\text { property islnputOf to instances of } \\
\text { ReputationEvaluationProcess class: } \exists \\
\text { isInputOf ReputationEvaluationProcess }\end{array}$ \\
\hline
\end{tabular}

1. Reasoning over the ontology in order to execute the subsumption test. The subsumption test determines whether there is a class in the ontology that subsumes another one, i.e., there is a class that is a superclass of the other. The superclass represents the semantic mapping of the original concept into the Functional Ontology of Reputation concept.

In our working example this step generates the classification of Regret system concepts in terms of the ontology classes. Table 2 shows these classifications. See also figure 11.

Table 2: Classification of ReGret Concepts

\begin{tabular}{|l|l|}
\hline Regret Concept & Concept classification \\
\hline Direct Trust & Primary Reputation \\
\hline Witness Reputation & Propagated Reputation \\
\hline $\begin{array}{l}\text { Neighbourhood Repu- } \\
\text { tation }\end{array}$ & Stereotyped Reputation \\
\hline System Reputation & Collective Reputation \\
\hline Credibility & Evaluation Factor \\
\hline
\end{tabular}

Figure 11 shows part of the asserted hierarchy as well as the inferred hierarchy generated in Protégé after the reasoning step.

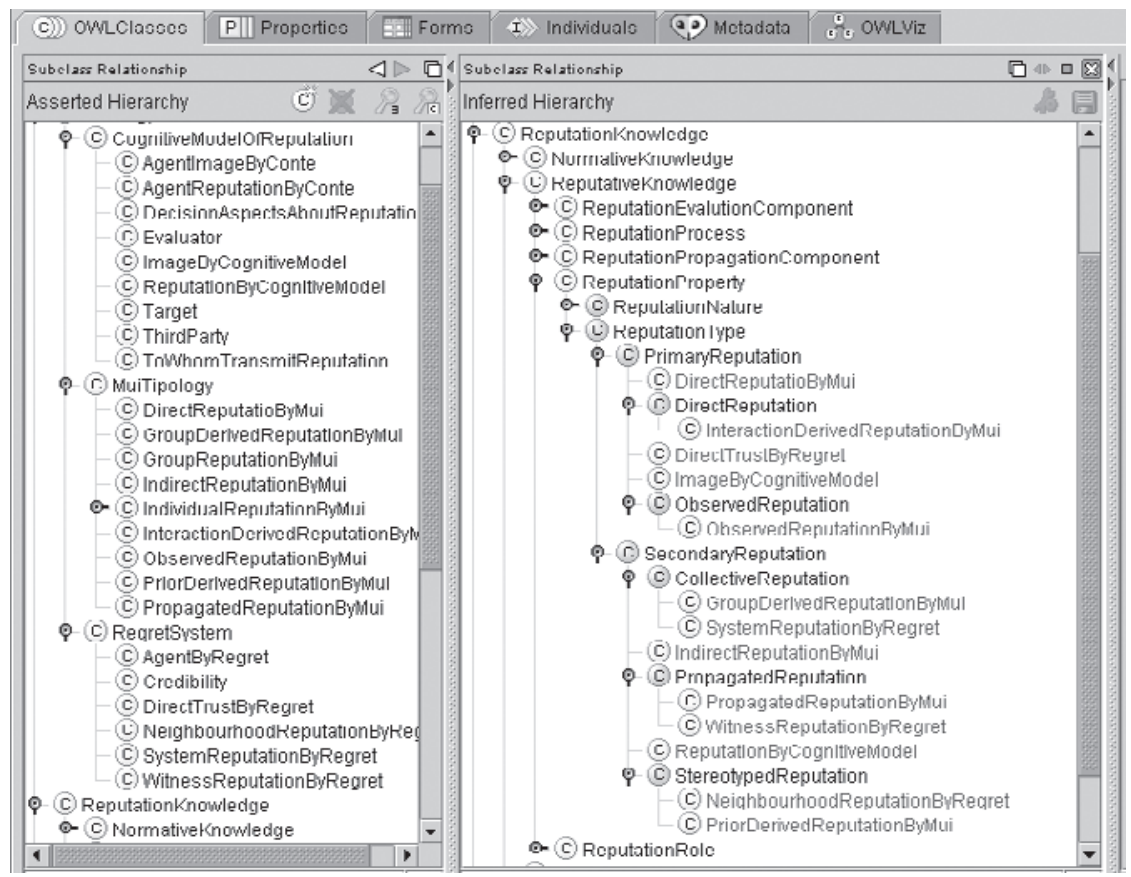

Figure 11: Protégé scream showing both asserted hierarchy and inferred hierarchy for classes involved in the semantic integration of reputation models. 
Table 3 highlights part of the semantic mapping results for the three reputation models. As we can see the classes related to reputation type in the Functional Ontology of Reputation act as a mapping between the three model concepts. For example, Direct Trust in ReGret system corresponds to Image in the Cognitive Reputation Model and to Direct Reputation in the Reputation Typology.

\begin{tabular}{|l|l|l|l|}
\hline $\begin{array}{c}\text { Functional } \\
\text { Ontology } \\
\text { Concept }\end{array}$ & $\begin{array}{c}\text { Cognitive } \\
\text { Model } \\
\text { Concept }\end{array}$ & $\begin{array}{c}\text { Reputa- } \\
\text { tion } \\
\text { Typology } \\
\text { Concept }\end{array}$ & $\begin{array}{c}\text { ReGret System } \\
\text { Concept }\end{array}$ \\
\hline $\begin{array}{l}\text { Primary } \\
\text { Reputation }\end{array}$ & Image & $\begin{array}{l}\text { Direct } \\
\text { Reputa- } \\
\text { tion }\end{array}$ & Direct Trust \\
\hline $\begin{array}{l}\text { Secondary } \\
\text { reputation }\end{array}$ & $\begin{array}{l}\text { Indirect } \\
\text { Reputa- } \\
\text { tion }\end{array}$ & Reputation \\
\hline $\begin{array}{l}\text { Propagated } \\
\text { Reputation }\end{array}$ & tion & $\begin{array}{l}\text { Propa- } \\
\text { gated } \\
\text { Reputa- } \\
\text { tion }\end{array}$ & $\begin{array}{l}\text { Witness Repu- } \\
\text { tation }\end{array}$ \\
\hline $\begin{array}{l}\text { Collective } \\
\text { Reputation }\end{array}$ & & $\begin{array}{l}\text { Group } \\
\text { Derived } \\
\text { Reputa- } \\
\text { tion }\end{array}$ & $\begin{array}{l}\text { System Repu- } \\
\text { tation } \\
\text { Ryped } \\
\text { Reputation }\end{array}$ \\
\hline
\end{tabular}

Table 3: Summary of semantic mapping results

\section{Discussion and Further Work}

In this paper we have presented some concepts used to identify reputation dimensions: reputation nature, roles involved in reputation formation and propagation, information sources for reputation, evaluation of reputation and reputation maintenance. Those concepts constitute a set of ontological terms that we have used to develop a Functional Ontology of Reputation. That ontology contains four main categories, distinguished by their function: Reputative Knowledge, Responsibility Knowledge, Normative Knowledge and World Knowledge. Besides, it includes the Common Knowledge category. These categories have been borrowed from or inspired by the Functional Ontology of Law proposed by Valente [29, 4].

The goal of this ontology is twofold. First, to put together, in a clear and coherent way, the broad knowledge about reputation disseminated in the literature. Second, to represent that knowledge in a structured form. That ontology has been implemented in a description logic language, the OWL- DL [11]. One of the key features of an ontology described in OWL DL is that we can reason over it, using a reasoner such as RACER [17] to process the ontology.
We claim that reputation knowledge structured as an ontology could be used to enable the semantic integration of agents using different reputation models. We have illustrated this idea using the Functional Ontology of Reputation as a common global ontology that supports the semantic integration among three reputation models, namely, the Cognitive Reputation Model [10], the Typology of Reputation [21] and the ReGret system [27].

As further work, we intend to extend the Functional Ontology of Reputation to be able to represent other notions closely related to reputation, like trust [9]. It could be a possible step toward the development of the trust layer in the context of the Semantic Web, as defined by Berners-Lee et al [1]. Additionally, the reasoning mechanism carried on by RACER [17] should be integrated in the semantic interoperation level in a middleware server and ontology alignment like PROMPT [22] and OBSERVER [20] should be incorporated in the solution in order to support the semantic integration level. Finally, we are evaluating the use of this ontology in the development of the Agent Reputation and Trust (ART) Testbed [14], an environment that aim to establish a testbed for agent trust and reputation-related technologies.

\section{ACKNowledgments}

This work has been developed as part of the project “A proposal for a Functional Ontology of Reputation for normative agents", supported by FAPESP, Brazil, grant number 04/15055-6. Jaime Simão Sichman was partially supported by CNPq, Brazil, grant numbers 304605/2004-2 and 482019/2004-2.

\section{REFERENCES}

[1] T. Berners-Lee; J. Hendler; O. Lassila. The Semantic Web. In: Scientific American, v. 284, n. 5, p. 28-37, 2001.

[2] O. Boissier ; Y. Demazeau. An architecture for social and individual control and its application to computer vision. In: Y. Demazeau; J.-P. Muller; J. Perram. (Ed.). Proceedings of the 6th European Workshop on Modelling Autonomous Agents in a Multi-Agent World, Odense, Denmark: [s.n.], p. 107-118, 1994.

[3] R. H. Bordini, J. F. Hübner. Jason: A Java-based agent speak interpreter used with saci for multi-agent distribution over the net, Available:http:// jason.sourceforge.net/.2004

[4] J.A. Breuker; A.Valente; R. Winkels. Legal ontologies: a functional view. In: P.R.S. Visser and R. Winkels, editors, Legal Ontologies, p. 23-36. ACM, New York. 1997.

[5] J. Breuker. Constructing a legal core ontology: LRICore. In: XVII Brazilian Symposium on Artificial 
Intelligence (SBIA). Workshop on Ontologies and their applications. São luiz do Maranhão, Brazil, 2004.

[6] D. B. Bromley. Reputation, Image and Impression Management. John Wiley \& Sons Ltd. England, 1993.

[7] S. Casare; J. S. Sichman. Towards a Functional Ontology of Reputation. In: Proceedings of 4th International Joint Conference on Autonomous Agents and Multi-agent Systems (AAMAS 2005), 2005.

[8] C. Castelfranchi; R. Conte; M. Paolucci. Normative reputation and the costs of compliance. In: Journal of Artificial Societies and Social Simulation vol. 1, no. 3, 1998.

[9] C. Castelfranchi; R. Falcone; G. Pezzulo. Trust in Information Sources as a Source for Trust: A Fuzzy Approach. In: Proceedings of 2nd International Joint Conference on Autonomous Agents and Multi-agent Systems (AAMAS'03), July 14-18, Melbourne, Australia, p. 89-96, 2003.

[10] R. Conte; M. Paolucci. Reputation in Artificial Societies: Social Beliefs for Social Order. Kluwer Academic Publishers, 2002.

[11] M. Dean; G. Schreiber. Editors. OWL Web Ontology Language Reference. W3C Recommendation, 10 February 2004. Available in: http://www.w3.org/TR/ 2004/REC-owl-ref-20040210/

[12] G. Ferris; F. Blass; C. Douglas; R. Kolodinsky; D. Treadway. Personal Reputation in Organizations. In: Greenberg, Jerald, Organizational Behavior- The State of the Science. LEA. New Jersey, p. 211-246, 2003.

[13] FIPA00001. 2000. FIPA Abstract Architecture Specification. Foundation for Intelligent Physical Agents. Available: http://www.fipa.org/specs/ fipa00001/.

[14] K. Fullam; T. B. Klos; G. Muller; J. Sabater; A. Schlosser; Z. Topol; S. Barber; J. S. Rosenschein; L. Vercouter; M. Voss. A specification of the Agent Reputation and Trust (ART) Testbed: Experimentation and Competition for Trust in Agent Societies. In: Proceedings of the Fourth International Joint Conference on Autonomous Agents and Multi-agent Systems (AAMAS 2005), p. 512-518, 2005.

[15] T. R. Gruber. Toward Principles for the Design of Ontologies Used for Knowledge Sharing. In: Nicola Guarino and Roberto Poli, editors, Formal Ontology in Conceptual Analysis and Knowledge Representation. Kluwer Academic Publishers, 1994

[16] N. Guarino. Formal Ontology, conceptual analysis and knowledge representation. In: International Journal of Human and Computer Studies, 43(5/6), p. 625-640, 1995.

[17] V. Haarslev; R. Moller. Racer: A core inference engine for the Semantic Web. In: 2nd International
Workshop on Evaluation of Ontology-based Tools (EON-2003), Sanibel Island, FL, 2003.

[18] K. Knublauch; M. A. Musen; A. L. Rector. Editing Description Logic Ontologies with the Protégé OWL Plugin. In: International Workshop on Description Logics - DL2004,Whistler, BC, Canada, 2004.

[19] Y. Labrou; T. Finin; Y. Peng. Agent communication languages: the current landscape.In: IEEE Intelligent Systems, v. 14, n. 2, p. 45-52, March/April, 1999.

[20] E. Mena; V. Kashyap; A. P. Sheth; A. Illarramendi. OBSERVER:AnApproach for Query Processing in Global Information Systems based on Interoperation across Preexisting Ontologies. In: Proceeding of the Conference on Cooperative Information Systems, p. 14-25,1996.

[21] L. Mui; A. Halberstadt; M. Mohtashemi. Notions of Reputation in Multi-Agents Systems: A Review. In: Proceedings of 1st International Joint Conference on Autonomous Agents and Multi-agent Systems (AAMAS 2002), Bologna, Italy, 2002.

[22] N. Noy; M. Musen. PROMPT: Algorithm and tool for automated ontology merging and alignment. In Seventeenth National Conference on Artificial Intelligence (AAAI-2000), Austin, TX, 2000.

[23] N. Noy; M. Musen. Using PROMPT OntologyComparison Tools in the EON Ontology Alignment Contest. In: Proceedings of the Third International Workshop on Evaluation of Ontology based Tools (EON 2004), 2003.

[24] A. S. Rao; M. P. Georgeff. BDI agents: from theory to practice. In: LESSER, V. (Ed.). International Conference on MultiAgent Systems (ICMAS'95), 1, San Francisco, USA, p. 312-319, 1995.

[25] J. Sabater; C. Sierra. Reputation and Social Network Analysis in Multi-Agent Systems. In: Proceedings of the first international joint conference on autonomous agents and multiagent systems (AAMAS02), Bologna, Italy. pp.475-482, 2002.

[26] J. Sabater; C. Sierra. Review on Computational Trust and Reputation Models. In: Artificial Intelligence Review, vol 24, n. 1, 2005.

[27] J. Sabater. Trust and reputation for agent societies. $\mathrm{PhD}$ Thesis. Institut d'Investigacion en Intelligencia Artificial, Spain, 2003.

[28] M. Uschold; M. Gruninger. Ontologies: Principles, Methods and Applications. In: Knowledge Engineering Review, v. 11, No. 2, June 1996.

[29] A. Valente. Legal Knowledge Engineering - A modeling Approach. IOS Press, Amsterdam, 1995.

[30] U. Visser; H. Stuckenschmidt; H. Wache; Thomas Vogele. Enabling technologies for interoperability. In: In U. Visser and H. Pundt, Editors, Workshop on the 14th International Symposium of Computer Science for Environmental Protection, p. 35-46, Bonn, Germany, 2000. 
[31] B. Yu; M. P. Singh. An Evidential Model of Distributed Reputation Management. In: Proceedings of 1st International Joint Conference on Autonomous Agents and Multi-agent Systems (AAMAS 2002), Bologna, Italy, 2002.

[32] G. Zacharia; P. Maes. Trust Management Through Reputation Mechanisms. In: Applied Artificial Intelligence, 14((9), Special Issue on Trust, Deception and Fraud in Agent Societies, p. 881-907, 2000. 